\section{Still waters run deep: latent cytokine activity in nonlesional psoriasis skin}

\author{
DOI: $10.1111 /$ bjd. 14248
}

Linked Article: Chiricozzi, et al. Br J Dermatol 2016; 174: 136-45.

Despite looking superficially unremarkable, the clinically uninvolved skin of patients with chronic plaque psoriasis has been shown to have a number of distinctions when compared with the skin of healthy control volunteers. The Koebner response, ${ }^{1}$ first described in 1876, as well as other studies on the abnormesverhalten or the abnormal behaviour of uninvolved skin, ${ }^{2}$ began to establish these differences but their underlying mechanisms could only be the subject of conjecture. Reports from the 1980s, making use of newly available monoclonal antibodies, found increased numbers of $\mathrm{CD}^{+}$and $\mathrm{CD}^{+} \mathrm{T}$ cells in the uninvolved skin of patients with guttate psoriasis, ${ }^{3}$ which gave an immunological basis to these discussions. Over the last 15 years, transcriptomic studies using microarrays, and later RNA-seq, have revealed a striking pattern of differential gene expression between lesional psoriasis skin and clinically uninvolved skin. ${ }^{4-7}$ However, fewer studies have focused on the differences between clinically uninvolved skin and the normal skin of unaffected control subjects. The first direct treatment of $\operatorname{such}^{6}$ revealed that while clinically uninvolved skin and healthy skin transcriptomes appeared to cluster together, setting a threshold to count all genes at least 1.3-fold altered in expression (with false discovery rate corrected P-value <0.05) identified 58 genes upregulated and 121 genes downregulated in uninvolved psoriatic skin. These 179 differentially expressed genes encoded proteins involved in epidermal differentiation, antimicrobial defences, lipid metabolism and regulation of cutaneous vasculature. These results identified a 'prepsoriatic' gene expression signature within uninvolved skin and pointed to decreased lipid biosynthesis and increased innate immunity in clinically uninvolved psoriatic skin. In this issue of the BJD, Chiricozzi and colleagues show that uninvolved psoriasis skin, distant from lesions, displays the molecular signature of interleukin (IL)-17 activity, in that there is elevated expression of genes downstream of IL-17, suggesting that the increased levels of IL-17 circulating in the blood of patients with psoriasis impacts nonlesional skin. Expanding on this, the authors use gene set enrichment analysis (GSEA), ${ }^{9}$ a powerful statistical tool to identify significantly enriched or underrepresented groups of genes within large datasets (e.g. cDNA microarrays, RNA-seq or proteomics datasets). In this instance, the authors show that within the transcriptome of nonlesional psoriasis skin hides the gene set upregulated in psoriasis lesions, as well as keratinocyte gene sets induced by the action of IL-17A, IL17A+TNF (tumour necrosis factor) $\alpha$ and IL-17A+IL-22, key cytokines in the pathogenesis of psoriasis. Interestingly, GSEA also detected the presence of T-helper (Th)1, Th17, Th22 and Th2 T-cell signatures in the nonlesional skin. This was likely a result of circulating cytokines acting on the uninvolved skin, but also could suggest the presence of resting resident memory $\mathrm{T}$ cells, particularly in resolved lesions; ${ }^{10}$ alternatively this 'residual disease genomic profile' could be the 'molecular scar' remaining in formerly lesional skin. ${ }^{11}$ Given these most recent observations and the development of multiomics studies harnessing transcriptomics, lipidomics, proteomics, epigenetics and genetics, we are approaching an era where we will be able to provide a full mechanistic rationale for the 'abnormal behaviour' of clinically uninvolved skin.

\section{Funding sources}

The authors acknowledge support from The National Institutes of Health grants AR064765 and AR060802 and the Babcock Memorial Trust.

\section{Conflicts of interest}

None declared.

${ }^{1}$ Department of Dermatology, University of Michigan, Ann Arbor, MI, U.S.A.

${ }^{2}$ Department of Dermatology, the First Affiliated Hospital of Nanjing Medical University, Nanjing, Jiangsu, China

E-mail: andjoh@med.umich.edu

\section{References}

1 Köbner H. Zur Aetiologie Psoriasis. Vjschr Dermatol 1876; 3:559.

2 Braun-Falco O, Christophers E, Kurban A. [Abnormal behavior of epidermal regeneration in patients with psoriasis vulgaris]. Arch Klin Exp Dermatol 1967; 229:276-84 [Article in German].

3 Baker BS, Swain AF, Fry L et al. Epidermal T lymphocytes and HLA-DR expression in psoriasis. Br J Dermatol 1984; 110:555-64.

4 Bowcock AM, Shannon W, Du F et al. Insights into psoriasis and other inflammatory diseases from large-scale gene expression studies. Hum Mol Genet 2001; 10:1793-805.

5 Zhou X, Krueger JG, Kao MC et al. Novel mechanisms of T-cell and dendritic cell activation revealed by profiling of psoriasis on the 63,100-element oligonucleotide array. Physiol Genom 2003; 13:69-78.

6 Gudjonsson JE, Ding J, Li X et al. Global gene expression analysis reveals evidence for decreased lipid biosynthesis and increased innate immunity in uninvolved psoriatic skin. J Invest Dermatol 2009; 129:2795-804.

7 Li B, Tsoi LC, Swindell WR et al. Transcriptome analysis of psoriasis in a large case-control sample: RNA-seq provides insights into disease mechanisms. J Invest Dermatol 2014; 134:1828-38.

8 Chiricozzi A, Suárez-Fariñas M, Fuentes-Duculan J et al. Increased expression of interleukin-17 pathway genes in nonlesional skin of moderate-to-severe psoriasis vulgaris. Br J Dermatol 2016; 174:13645 .

9 Subramanian A, Tamayo P, Mootha VK et al. Gene set enrichment analysis: a knowledge-based approach for interpreting genome- 
wide expression profiles. Proc Natl Acad Sci USA 2005; 102: $15545-50$.

10 Cheuk S, Wiken M, Blomqvist L et al. Epidermal Th22 and Tc17 cells form a localized disease memory in clinically healed psoriasis. J Immunol 2014; 192:3111-20.

11 Suarez-Farinas M, Fuentes-Duculan J, Lowes MA et al. Resolved psoriasis lesions retain expression of a subset of disease-related genes. J Invest Dermatol 2011; 131:391-400.

\section{Prognostic and predictive biomarkers for the benefit of immunotherapy in patients with metastatic melanoma}

\author{
DOI: $10.1111 /$ bjd.14251
}

\section{Linked Article: Zaragoza, et al. Br J Dermatol 2016; 174: 146-51}

The treatment of patients with metastatic melanoma has been a major therapeutic challenge for decades, and only recently has significant progress in the treatment of the disease been made. In the last 4-5 years, new targeted therapies such as BRAF and mitogen-activated protein kinase (MEK) inhibitors have been introduced into the armamentarium for patients whose melanoma harbours the V600 BRAF mutation. In addition, we have seen a significant expansion in the field of immunotherapy: ipilimumab, cytotoxic T-lymphocyte-associated protein 4 blocking antibody, pembrolizumab and nivolumab, and programmed cell death protein 1 blocking antibodies have joined high-dose interleukin 2 as therapeutic options.

Ipilimumab was approved based on the results of a phase III trial in which patients were randomized to ipilimumab alone, ipilimumab in combination with gp100 vaccine and gp100 vaccine alone. ${ }^{1}$ Despite a low response rate of $11 \%$, treatment with ipilimumab led to a near 4-month improvement in overall survival $(10 \cdot 1$ months in the ipilimumab arm and 6.4 months in the gp100 arm). The recently published data on the follow-up of 1861 patients treated with ipilimumab show a plateau, starting at 3 years, in the survival curve at $21 \%{ }^{2}$ This long-term benefit of the therapy made ipilimumab an attractive therapeutic option; however, as only a minority of patients benefit, a significant effort has been put in the search of predictive and prognostic biomarkers.

The article by Zaragoza et al. in this issue of the BJD describes the analysis of the outcome of 58 consecutive patients treated with ipilimumab in a single institution. ${ }^{3}$ The authors show that patients with a neutrophil-to-lymphocyte ratio $(\mathrm{NLR})>4$ had a significantly shortened survival. Caution must be exercised if attempting to use these results in clinical practice. Did the authors study a prognostic or a predictive biomarker? Prognostic biomarkers correlate with the natural progression or aggressiveness of a disease and are used to estimate median survival. Predictive biomarkers are used to estimate probability for a response to a given treatment, and therefore they are especially valuable when assessed before the treatment is initiated. ${ }^{4}$ When participants are not randomly assigned to an intervention it is nearly impossible to assess the impact of this intervention on the outcome. In this research, the authors measured a biomarker (NLR) before the intervention (treatment with ipilimumab), but they did not randomize patients to a 'treatment' or 'no treatment' group. Therefore, the results tell us nothing about how predictive NLR is for response to the treatment, but they do tell us that NLR has a prognostic value, that is, the worse prognosis when NLR is elevated will not be overcome with the use of ipilimumab. I strongly discourage clinicians to use the NLR when they make a decision on the choice of the therapy, but I encourage them to use it as a part of the discussion with patients on their prognosis.

To date, no true predictive biomarkers for the response to therapy with ipilimumab have been identified. The findings supporting the presence of germline genetic factors associated with response to ipilimumab therapy are especially intriguing. ${ }^{5}$ In order to confirm the validity of this discovery a larger number of samples would have to be analysed and the findings would require validation in an independent cohort.

\section{Conflicts of interest}

The author was on the Advisory Board and Speaker Bureau for Bristol Myers Squibb.

Division of Hematology - Medical Oncology,

B. Chmielowski

Jonsson Comprehensive Cancer Center,

University of California Los Angeles, Los

Angeles, CA 90049, U.S.A.

E-mail: bchmielowski@mednet.ucla.edu

ORCID: https://orcid.0000-0002-2374-

3320

\section{References}

1 Hodi FS, O’Day SJ, McDermott DF et al. Improved survival with ipilimumab in patients with metastatic melanoma. N Engl J Med 2010; 363:711-23.

2 Schadendorf D, Hodi FS, Robert C et al. Pooled analysis of long-term survival data from phase II and phase III trials of ipilimumab in unresectable or metastatic melanoma. J Clin Oncol 2015; 33:1889-94.

3 Mahoney KM, Atkins MB. Prognostic and predictive markers for the new immunotherapies. Oncology (Williston Park) 2014; 28(Suppl. 3):39-48.

4 Zaragoza J, Caille A, Beneton N et al. High neutrophil to lymphocyte ratio measured before starting ipilimumab treatment is associated with reduced overall survival in patients with melanoma. Br J Dermatol 2016; 174:146-51.

5 Adaniel C, Rendleman J, Polsky D et al. Germline genetic determinants of immunotherapy response in metastatic melanoma. ASCO Meeting Abstracts 2014; 32:3004. 